\title{
Yod
}

Revue des études hébraïques et juives

$14 \mid 2009$

La littérature israélienne, miroir d'une société multiple

\section{Les prémices de la littérature hébraïque moderne en Irak et son passage en Israël}

Hebrew Literature in Modern Iraq

ספרות עברית מודרנית בעיראק

Lev Hakak

\section{OpenEdition}

Journals

Édition électronique

URL : https://journals.openedition.org/yod/348

DOI : $10.4000 /$ yod. 348

ISSN : 2261-0200

Éditeur

INALCO

Édition imprimée

Date de publication : 1 octobre 2009

Pagination : 105-118

ISBN : 978-2-85831-178-1

ISSN : 0338-9316

\section{Référence électronique}

Lev Hakak, « Les prémices de la littérature hébraïque moderne en Irak et son passage en Israël », Yod [En ligne], 14 | 2009, mis en ligne le 31 octobre 2011, consulté le 08 juillet 2021. URL : http:// journals.openedition.org/yod/348; DOI : https://doi.org/10.4000/yod.348

Ce document a été généré automatiquement le 8 juillet 2021.

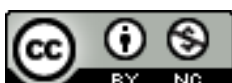

Yod est mis à disposition selon les termes de la Licence Creative Commons Attribution - Pas d'Utilisation Commerciale 4.0 International. 


\title{
Les prémices de la littérature hébraïque moderne en Irak et son passage en Israël
}

\author{
Hebrew Literature in Modern Iraq
}

ספרות עברית מודרנית בעיראק

\author{
Lev Hakak
}

\section{La littérature hébraïque moderne en Irak de 1735 à 1950}

1 L'histoire de la littérature hébraïque moderne ne tient pas compte de l'œuvre hébraïque irakienne. Sans doute, est-ce dû au fait qu'il ne reste presque rien des piyyutim irakiens du quinzième siècle à la première moitié du dix-neuvième siècle. Cependant, à partir de cette époque jusque dans les années 1951-52, date où les cent vingt mille juifs émigrèrent en Israël au cours de l'opération « Ezra et Néhémie », existe une œuvre littéraire prolifique qui contient entre autres plus de deux mille piyyutim et poèmes dont la plupart sont des poésies sacrées et quelques poésies profanes. Ils nous permettent d'étudier leur style et de définir leurs lecteurs ainsi que l'esprit et le poète. Les thèmes de la majeure partie de l'œuvre écrite en Irak portent sur la religion et la Halakha. Une poésie profane est aussi présente : les Juifs d'Irak écrivaient en hébreu des épîtres, des histoires courtes, des spectacles, des études littéraires, des contes folkloriques, etc.

2 La poésie hébraïque d'Irak fut influencée par la poésie hébraïque en Espagne, ainsi que par la poésie hébraïque de son époque. La poésie en Espagne influença une partie des poètes par ses thèmes, sa poétique, la construction des strophes (deux hémistiches) la métrique, la versification, la langue créative, l'encastrement des versets, etc. Cependant, la poésie hébraïque d'Irak fut également influencée par la poésie de son époque. On peut compter parmi les «conduits» influents de cette littérature, les 
voyageurs et travailleurs temporaires partis d'Irak vers l'Europe et vice-versa, des professeurs venus d'Europe et d'Eretz Israël, des juifs irakiens abonnés aux publications hébraïques en Europe et en Eretz Israël. Les Juifs d'Irak publièrent eux-mêmes à différentes époques des journaux en hébreu qui contenaient des informations et des analyses littéraires. Les écoles modernes qui s'ouvrirent en Irak établirent des ponts entre l'Orient et l'Occident. À partir de 1863 plus de cinq cents livres en hébreu furent publiés en Irak.

3 Quelques exemples permettront d'illustrer la diffusion de l'hébreu en Irak durant cette période. Les institutions scolaires juives en Irak -- des jardins d'enfants aux écoles rabbiniques -- insistaient sur l'enseignement de l'hébreu. À partir de 1832, celui-ci était dispensé au heder et dans les classes de Talmud-Torah où l'on enseignait l'hébreu et la Bible, de l'apprentissage de la lecture à la traduction en judéo-arabe des Prophètes et des Hagiographes et à l'enseignement du Talmud. En 1840, année où fut fondée l'école rabbinique "Bet Zilca », il existait à Bagdad des dizaines de hadarim. Fin 1864, une école de l'Alliance israélite universelle ouvrit ses portes à Bagdad. Dans les années 1925-1935, des professeurs venus d'Erets Israël enseignaient à Bagdad. En 1924, le « Maître » Aaron Sasson fonda "Pardes ha-yeladim », une école où l'enseignement était dispensé en hébreu et qui fut fermée en 1935, quand le « Maître » fut obligé de quitter Bagdad en raison de ses idées sionistes. On montait dans cette école des pièces de théâtre en hébreu, on y tenait des conférences en hébreu et on y trouvait l'une des bibliothèques hébraïques de Bagdad. Abraham Rozen, qui vint d'Erets Israël pour enseigner en Irak, décrit en 1932 une communauté dans laquelle l'hébreu est répandu et compris par presque tous les enfants juifs. Jusqu'en 1912, on utilisa également la Bible pour enseigner l'hébreu, ce qui explique la connaissance approfondie de la Bible par les écrivains hébraïques irakiens.

Un exemple intéressant de la création littéraire hébraïque en Irak de 1735 à 1950 est le livre de poésie moraliste, Tokhehot musar, publié en 1735 par Ezra Habavli et dans lequel l'auteur, sur environ 225 pages, admoneste ses lecteurs. Ce livre témoigne d'une langue riche, créative, d'une imagination foisonnante, d'une capacité d'illustration impressionnante, d'une âme impétueuse et sensible.

5 La poésie de Habavli n'est pas inférieure à celle de Moshe Hayyim Luzzatto (1707-1747) non seulement si l'on considère le petit recueil de poèmes de ce dernier Elle bne hane urim (1768), mais aussi les pièces en vers qui lui valurent des louanges, Migdal 'oz (1738), Ma'ase Shimshon (1728) et La-yesharim tehilla (1743). Il me semble que la qualité esthétique des textes poétiques ne suffit pas à elle seule à expliquer pourquoi nous n'avons pas entendu parler d'Ezra Habavli, alors que P. Lahover voit dans l'œuvre de Luzzatto le début de la littérature hébraïque moderne.

6 La prose hébraïque en Irak n'est pas riche, mais existe néanmoins. Le rabbin Shlomo Bekhor Hotsin (1843-1892) a publié dans les revues Ha-Maggid, Ha-Levanon, Ha-Tsfira, Ha-'Ivri, Ha-Melits environ cent cinquante notices sur la vie des Juifs en Irak, en Iran et au Kurdistan. Il a également écrit des piyyutim, des articles sur le judaïsme, l'éducation. Traducteur, pédagogue, homme public, il a aussi fondé une imprimerie hébraïque. Ses notices ont une importance historique, culturelle et linguistique.

7 Sha'ul Abdallah Yossef (1849-1906), un natif de Bagdad installé à Hong-Kong, était poète, exégète biblique et spécialiste de la poésie hébraïque en Espagne qu'il a été, avec David Yelin, le premier à analyser méthodiquement. Sha'ul Yossef a publié des articles sur cette poésie dans Ha-Tsfira, ainsi que dans Perah et Maggid mesharim (publiés en 
Inde). Il a fait paraitre ses commentaires sur les poèmes de Moïse Ibn Ezra et édité Gan ha-meshalim we-ha-hidot de Todros Abulafia, ouvrage qu'il a découvert. Il a cherché à apporter sa contribution à l'élaboration d'une version fiable de la poésie hébraïque médiévale et des commentaires sur celle-ci. Il avait le sentiment que cette poésie le touchait personnellement et il a critiqué chercheurs et commentateurs de sa génération.

8 Pour certains, c'est le rabbin et poète Suliman Mani (1850-1924) qui ouvre la voie de la prose hébraïque moderne chez les Juifs d'Orient avec sa nouvelle 'Emeq ha-siddim.

Rappelons qu'il y avait en Irak des périodiques en hébreu: Ha-Dover (1870-1871), Yeshurun (1921), Shemesh (1930-1933) et Derekh he-haluts (1945-1951). Yeshurun se définissait comme un journal littéraire, social et historique et ses cinq numéros comportent des poèmes et des articles. Derekh he-hialuts a publié des notices, des poèmes, des pièces de théâtre et des articles de dirigeants du mouvement He-haluts. Shemesh était un journal publié par des élèves du primaire et du collège. Derekh hehaluts et Shemesh témoignent de l'interaction avec la littérature hébraïque de l'époque.

\section{Le passage en Israël}

10 Il y avait donc en Irak, au moment du départ des Juifs pour Israël en 1951-1952, des gens sachant l'hébreu, écrivant en hébreu. Cependant, la création littéraire des Juifs originaires d'Irak en Israël n'est pas la continuation de la création hébraïque en Irak. Les auteurs qui écrivaient en hébreu en Irak, y compris ceux qui ont continué d'écrire en hébreu en Israël, ne se sont pas intégrés dans la communauté littéraire israélienne. Il s'agit d'écrivains comme Aaron Sasson, (Irak 1877 - Jérusalem 1962) qui, avant d'immigrer en Palestine en 1936, a publié un recueil de poèmes à Bagdad en 1925; David Hay Abboudi qui a publié en 1930 un recueil de ses poèmes en hébreu et a immigré en Israël en 1951; Shlomo ben Salah Shlomo (Irak 1896 - Tel Aviv 1961, immigré en 1951) qui a publié en Irak en 1925 un recueil de poésie; Menasheh Shahrabani (1881-1960, arrivé en Israël en 1950) qui a publié deux recueils de poèmes à Bagdad et a continué à écrire en Israël ; David Tsemah (1902-1981, arrivé en 1949) ; Ezra Naji Mkamal (1912-2000) et d'autres encore. Comment et pourquoi cela s'est-il produit? Comment se fait-il que des écrivains hébraïques venus d'Europe en Israël s'y soient fait une place dans la vie littéraire, ont crû et prospéré dans un lieu où l'on parlait l'hébreu et où ils pouvaient avoir un public relativement nombreux, alors que les écrivains hébraïques venus d'Irak semblent n'avoir pas existé?

11 Il me semble qu'il faudrait avancer plusieurs raisons, certaines liées directement aux écrivains eux-mêmes et d'autres, à ceux qui étaient déjà installés en Israël. Les raisons que je vais exposer ne seront pas admises par tous et certaines ne s'appliquent pas à tous ces écrivains. Il ne s'agit pas d'un groupe important d'auteurs qui aurait occupé le devant de la scène si seulement ils avaient intégré l'univers littéraire à l'époque de leur alyah. Certains utilisaient des formes et un style passés de mode en Israël. La plupart sont arrivés en Israël dans les années cinquante, années difficiles qui n'ont laissé que peu de place à l'accomplissement intellectuel et ils ont perdu toute énergie dans les ma'barot. Pourtant, les auteurs qui ont immigré d'Europe vers Israël n'ont-ils pas souffert de difficultés matérielles ? Certes, mais l'interaction qui s'est établie entre eux, leur sentiment d'appartenance et leur intégration leur ont insufflé énergie et courage et leur ont permis de se consacrer, plus que les écrivains venus d'Irak, à leur écriture. 
De même, il me semble que les écrivains influents de cette époque n'étaient pas préparés à accueillir les écrivains hébraïques récemment arrivés d'Irak. Ceux-ci possédaient une culture différente de la dynamique sociale et manquaient de cette expérience qui leur aurait permis de se rapprocher de la société littéraire et de s'y intégrer. L'ouverture et la conscience du pluralisme social et culturel en Israël ont été le résultat d'un long processus.

David ben Suliman Tsemah (1902), par exemple, qui a écrit des poèmes proches de l'esprit de sa génération, il s'est rendu en 1931 et 1935 en Palestine où il s'est lié d'amitié avec Bialik et Hayyim Brodi et pourtant, il n'a sa place dans notre histoire littéraire. Il en est de même pour Aaron Sasson qui a été d'un courage extrême au cours d'un procès condamnant ses activités sionistes. Craignant le pouvoir irakien, il a immigré en 1936 et lui non plus n'a pas eu la place qu'il méritait, ni dans le domaine de la littérature - ses œuvres n'étant pas au goût des lecteurs de l'époque - ni même au sein des institutions sionistes auxquelles il a consacré sa vie.

Les écrivains originaires d'Irak connus aujourd'hui sont ceux qui ont commencé à écrire en hébreu en Israël. Il est peut-être possible de mettre un terme à l'idée erronée qu'il n'y a jamais eu de littérature hébraïque moderne en Irak avant celle qui s'est développée en Israël, en quelque sorte ex nihilo. Pour ce faire, il faudrait mettre en lumière la culture hébraïque en Irak. La documentation, la description, l'évaluation et l'analyse de l'œuvre des Juifs orientaux nous donneraient un tableau historique plus varié de la littérature hébraïque moderne. Même si ce tableau ne mène pas à de grandes découvertes sur le plan des qualités littéraires, il permettra d'inclure certains auteurs dans le canon littéraire moderne, à l'image de ceux qui en font partie aujourd'hui et qui ne sont pas tous doués d'un grand talent.

Les premiers auteurs d'origine irakienne à publier en hébreu en Israël avaient pour langue maternelle l'arabe, puis sont apparus ceux dont la langue maternelle a été l'hébreu. Shlomo Zamir, Shalom Katav et Aaron Zakaï, par exemple, ont d'abord écrit en arabe puis sont passés à l'hébreu. Zakaï et Zamir ont publié leurs premiers recueils de poésie peu de temps après leur arrivée en Israël, Zakaï en 1957 et Zamir en 1960. Des poètes venus d'Europe ont eux aussi fait l'expérience du passage à l'hébreu : je pense par exemple à Rahel et Ezra Zussman qui ont d'abord écrit en russe, Shin Shalom - en allemand, Anda Amir Finkerfeld - en polonais. Herzl et Balfour Hakak sont arrivés en Israël à l'âge de deux ans et écrivent en hébreu. Les auteurs venus d'Irak qui ont continué d'écrire en arabe n'ont eu qu'un public restreint. Shmuel Moreh, Sasson Somekh et David Tsemah sont passés de l'écriture poétique en arabe à la recherche littéraire.

On ignore donc qu'il y a continuité de la création littéraire en hébreu et une génération d'écrivains qui a commencé à publier en hébreu en Irak pour continuer ensuite en Israël.

\section{La littérature hébraïque des auteurs d'origine irakienne en Israël}

La communauté des Juifs originaires d'Irak est l'une des plus importantes d'Israël et elle a donné naissance à un certain nombre d'écrivains connus d'un large public. 
17 Dans le domaine de la poésie, il faut distinguer entre, d'une part, les poètes dont l'hébreu a été la première langue apprise à l'école parce qu'ils sont arrivés en Israël dans leur enfance ou qu'ils y sont nés et d'autre part, les poètes plus âgés. On peut ainsi compter dans le premier groupe Herzl et Balfour Hakak, Tsvi Hakak, Lev Hakak, Yossi Ozer, Haviva Ovadya-Pedaya, Liliane Debi-Gouri, Tikvah Shukr, Yossi Alfi, Zamir BenDavid, Amira Hess, Yaara Ben-David, Yaacov Yaacov et Roni Someck. Herzl et Balfour Hakak, par exemple, sont nés en Irak en 1948 et sont arrivés en Israël à l'âge de deux ans seulement; ils ont publié des recueils de poésie à partir de 1970. Le poète Roni Someck, né en Irak en 1951, est arrivé en Israël en 1953 et a publié son premier livre de poésie en 1976.

Le second groupe est composé de poètes nés en Irak dans les années vingt et trente du siècle dernier par exemple: Aaron Zakaï, Shlomo Zamir, Yoav Hayek, Lili Zalouf, Clarisse Dayan (née en 1936), Shalom Katav (Bagdad 1931), Emile Mourad, Tsion Matitiahou, David Rabi, Yehezkel Moriel, Yitshak Horesh, Ilana Rotman, Jacqueline Shashoua Gabay qui a également publié de la prose, Yaacov Shaya. Ces poètes ont publié en hébreu à partir des années soixante.

19 Shalom Katav a d'abord écrit des poèmes en arabe. Son premier recueil de poésie en hébreu, Lo rash me'od ("Pas si pauvre»), a paru en 1972 et le second, 'Al gedot hanaharayim ("Sur les rives des deux fleuves»), un an plus tard. Le premier recueil de poèmes publié par un poète d'origine irakienne en Israël est 'Al hofo shel ra'yon (« Au bord d'une idée ») d'Aaron Zakaï en 1957. Trois ans plus tard, Shlomo Zamir a publié Ha-qol mi-ba'ad le-'anaf («La voix à travers une branche»). Je n'ai pas l'intention de classer ces auteurs selon des critères esthétiques, mais je soulignerais simplement que la poésie de Shlomo Zamir est à mes yeux digne de trouver une place dans notre canon littéraire. La publication de son nouvel ouvrage qui reprendra les poèmes des trois volumes déjà publiés auxquels viendront s'ajouter trois nouveaux recueils y conduira peut-être.

20 Ces poètes ne se distinguent pas par les thèmes qu'ils abordent. De même que les auteurs plus âgés ont traité de sujets ancrés dans le pays où ils ont immigré, de même ceux arrivés en Israël pendant l'enfance ont-ils abordé des thèmes enracinés en Irak. Ainsi, Shalom Katav ne s'est pas polarisé dans son premier livre de poèmes sur des thèmes qui expriment son identité spécifique, alors que dans le recueil suivant 'Al gedot ha-naharayim, il a réuni des poésies sur la vie des juifs en Irak et a même décrit les émeutes antijuives de 1941. Il en est de même pour les jumeaux Herzl et Balfour Hakak qui ont écrit aussi des poèmes sans rapport avec leur origine, mais ont publié, chacun par la suite, un recueil de poésie sur ces émeutes (1987).

21 Pour ce qui concerne le roman, le premier thème chez les auteurs originaires d'Irak est le traumatisme de la rencontre avec la terre d'Israël, thème vite délaissé au profit d'autres.

La génération des premiers auteurs qui ont immigré d'Irak a d'abord écrit sur l'expérience de l'arrivée en Israël, sur les tensions intercommunautaires puis sur la vie en Irak et en Israël, sur les Arabes et sur les relations entre Juifs et Arabes. Les premiers romans combattent les stéréotypes, considérés comme le fruit d'une erreur, d'un sentiment d'arrogance ou de tendances politiques. Ce sont des romans réalistes qui exposent d'âpres confrontations entre des personnages d'origine irakienne et d'autres. Puis ont été abordés le mode de vie en Irak et en Israël et les rapports entre Juifs et Arabes. 

(1964), Shawim we-shawim yoter («Tous sont égaux... mais certains le sont plus») de Sammy Michael (1974), Kapak ha-bagdadi («Kapak de Bagdad») de David Rabi (1975), Ha-asufim («Les enfants trouvés ») de Lev Hakak (1977) et Tarnegol kapparot (« Le coq expiatoire ») (1983) d'Eli Amir. Ces romans ont pour toile de fond la grande alyah de 1950-1951 et la vie des Juifs irakiens dans les ma'barot. Ils décrivent le passage d'une vie confortable à une réalité économique et sociale difficile, l'écroulement des valeurs et l'humiliation ressentie. Ils expriment aussi la frustration, l'aliénation, les affronts, les privations, la misère et la discrimination. Le point de vue est celui de narrateurs, à la fois héros et témoins, qui décrivent, sur fond de tensions intercommunautaires, des personnages en conflit avec d'autres et aboutissent à des situations inextricables. travers l'exemple des romans de Sammy Michael (né en 1926). À la suite du roman contestataire Shawim we-shawim yoter, Michael a écrit Hasut («Refuge», 1977), roman centré sur les problèmes psychologiques et sociaux sur fond de conflit arabo-juif et d'idéologie du parti communiste israélien. Michael a ensuite publié $\underline{H}$ ofen shel 'arafel («Une poignée de brouillard», 1979) dans lequel il raconte, en y mêlant des éléments de la vie des Juifs et des Arabes en Irak, l'histoire de militants sionistes clandestins, de révolutionnaires arabes et juifs membres d'un mouvement communiste interdit à une époque marquée par l'agitation politique, à la veille de l'émigration des Juifs irakiens vers Israël. En 1987, Michael a publié $\underline{H}$ atsotsra ba-wadi (« Une trompette dans le wadi ", traduit en français en 2006) dans lequel les personnages principaux sont Huda, une jeune Arabe chrétienne, et Alex, un nouvel immigrant juif de Russie. Alex vit en marge de la communauté ashkénaze, il est fort, sensible, courageux et sa pauvre vie s'achève lors de la guerre du Liban. Victoria (1993, traduction française en 1998) marque un tournant en ce que, pour la première fois, un auteur d'origine irakienne décrit la vie de sa communauté dans un passé lointain, du début du XIX siècle à Bagdad jusqu'aux années quatre-vingt en Israël. Sammy Michael a accompagné la parution de son œuvre de déclarations contradictoires. Son roman a d'abord été présenté comme autobiographique, puis comme un roman décrivant les couches les plus démunies de la communauté juive d'Irak, puis comme un roman sur la misère des Juifs irakiens à la fin, uniquement, de l'époque ottomane. Pour finir, Michael a déclaré que son livre présentait la vie de la communauté durant toute cette époque. De fait, ce roman contient nombre d'éléments autobiographiques. Ensuite ont été publiés Mayim noshqim le-mayim («L'eau touche l'eau », 2001), un roman sur fond des années cinquante en Israël et sur l'amour d'un immigrant d'Irak d'abord pour une femme meurtrie puis une autre pleine de vie, et Yonim be-Trafalgar ("Colombes à Trafalgar », 2005), un livre dans lequel il rend hommage à la maternité, que ce soit chez les Juifs ou chez les Arabes, et décrit les rapports entre Israéliens et Palestiniens, sans se focaliser sur la culpabilité ou l'innocence des uns ou des autres, mais sur le caractère tragique de ces rapports. Aïda (2008) est une histoire d'amour entre Zaki, un vieil homme, l'un des derniers Juifs de Bagdad et Aïda, une rescapée kurde. Ce livre, comme ses autres romans, contient un discours politique.

Eli Amir ne s'est pas attardé non plus sur le sujet des tensions intercommunautaires. Neuf ans après Tarnegol kapparot, Amir décrit dans Mafriah ha-yonim ("Le colombier ", 1992) la vie en Irak, en particulier après la guerre de 1948 qui a eu pour effet l'écrasement de la fierté arabe et les persécutions infligées aux Juifs qui ont poussé ces 
derniers à trouver une voie de sortie. La dernière partie se déroule en Israël, la détresse conduit à la folie du père, toutefois lorsque son fils nait, une étincelle d'espoir semble luire dans ses yeux. Dans Ahavat Sha'ul («L'amour de Saül», 1998), Amir aborde à nouveau les tensions intercommunautaires en décrivant le double amour d'un jeune Séfarade, dont la famille vit en Terre d'Israël depuis sept générations, pour Israël et pour une fille de rescapés de la Shoah. Cet amour se termine par une déception et un rejet dû à son origine et à sa situation sociale, ce qui détermine la destinée du héros. Pgisha 'iweret ("Blind Date», 2000) est une nouvelle sur des hommes et des femmes en quête d'amour et une histoire d'amour entre une rescapée de la Shoah et un Israélien. Dans Yasmin (2005), le lecteur se trouve à la fin de la guerre des Six Jours, lorsque, dans les faits, Juifs et Arabes s'observent. Le livre soulève le problème de la conquête et la perspective d'une possible entente entre les deux peuples. Amir a donc commencé par des sujets portant sur les affrontements sociaux et culturels en Israël, la culture des immigrants et leurs difficultés d'intégration, puis il est passé au conflit arabo-juif en général et au conflit israélo-palestinien en particulier et il a essayé de présenter une position collective.

On peut donc dire qu'en règle générale, les auteurs d'origine irakienne ont produit des romans réalistes, les premiers polarisés sur les tensions intercommunautaires, les suivants ayant pour thème la vie de Juifs en Irak et les relations entre Juifs et Arabes. Les tensions intercommunautaires, la réalité en Irak comme cadre de l'intrigue, la réalité israélienne, les relations entre Juifs et Arabes reviennent dans quelques-uns de ces romans.

Dans leurs nouvelles, les écrivains originaires d'Irak mettent aussi l'accent sur les tensions intercommunautaires et témoignent du mode de vie, comme, par exemple, Eliyahou Patael dans son recueil Be-tsel ha-tshuqa («A l'ombre de la passion », 1965), Nir Shohat dans Ben or le-hoshekh ("Entre la lumière et l'obscurité », 1971) et dans 'Od layla («Encore une nuit »,1972). C'est aussi la règle dans les nouvelles de Shimon Ballas et David Rabi.

En conclusion, on peut dire que d'une manière générale, les romans des auteurs israéliens originaires d'Irak sont des romans réalistes qui tentent de reproduire la vie quotidienne, de décrire d'une manière plausible un monde fictif dans lequel prennent place des événements et des actions semblables à ceux de la vie réelle. Il ne faut pas y chercher des symboles à la signification difficilement accessible, des caractéristiques surréalistes, une ironie sous-jacente, une syntaxe novatrice unique, des images poétiques compliquées, des allusions sophistiquées aux textes anciens ou nouveaux. Ces allusions aux sources sont quasiment absentes, en particulier dans l'œuvre de la génération qui a fait la majeure partie de ses études en Irak.

Dans ces œuvres, les auteurs mêlent des événements et des personnages de l'histoire des Juifs irakiens, par exemple le Farhud ${ }^{1}$, la pendaison de Shafik Adas, la chanteuse juive Salima (Eli Amir), etc. et l'arabe et l'hébreu s'y trouvent souvent entremêlés.

30 Ces œuvres expriment l'idée qu'en ce qui concerne les Juifs orientaux, il existe un écart entre les préjugés et la réalité. La description des Israéliens déjà installés au moment de l'arrivée des Juifs d'Irak devient avec le temps plus complexe, modérée, favorable et équilibrée. L'univers décrit dans les œuvres qui ont pour toile de fond la vie en Irak est nouveau pour le lecteur. Ce qui prédomine, ce sont les problèmes idéologiques et sociaux dans lesquels s'entremêlent des histoires d'amour. Mais l'univers où cela se 
passe est éventuellement instable et il y est difficile, voire impossible, de concrétiser cet amour.

Les Juifs irakiens ont aussi publié des mémoires, œuvres importantes tant sur le plan littéraire qu'historique, comme, par exemple, celles de Salim Patael et Sasson Somekh. Je rappellerais par souci d'objectivité que l'auteur de ce texte a publié deux romans, un recueil de nouvelles et un recueil de poèmes.

Il me semble que l'on manque parfois de perspective pour apprécier une partie des auteurs cités et que le débat sur la qualité littéraire passe parfois après les thèmes qui sont nouveaux pour les lecteurs. Les positions sociales et l'intervention de l'auteur dans la promotion de son œuvre se mêlent au jugement littéraire. Il n'existe à ce jour aucun écrivain d'origine irakienne dont on s'accorde à dire qu'il a la valeur des grands auteurs hébraïques d'origine européenne ou nés en Israël. Il me semble pourtant qu'il en existe quelques-uns. Cependant, ces dernières années le tissu culturel et social de l'État d'Israël se modifie et la réception des auteurs de toutes communautés et origines est en train de se faire beaucoup plus facilement pour qu'ils trouvent enfin leur place dans un univers littéraire riche et varié.

\section{BIBLIOGRAPHIE}

BEN YAAKOV Avraham, Shira u-fiyyut shel Yehude Bavel ba-dorot ha-aharonim ( Poésie et piyyut des Juifs d'Irak à l'époque moderne »), Institut Ben-Zvi, 1970.

HAKAK Lev, Nitsane ha-yetsira ha-'ivrit ha-hadasha be-Bavel (« Les débuts de la littérature hébraïque moderne en Irak »), Merkaz moreshet yahdut Bavel, 2003 (voir la bibliographie qui comporte les références d'articles et d'ouvrages de l'auteur, dans lesquels il est question de l'œuvre des écrivains israéliens originaires d'Irak).

HAKAK Lev, Iggrot ha-rav Shlomo Bekhor Hotsin («Épîtres du rabbin Shlomo Bekhor Hotsin »), Hakibbutz Hameuchad, Collection Helal Ben Haïm, 2005.

HAKAK Lev, Hasid mul hot'im - sefer ha-tokhahot shel 'Ezra Ha-Bavli (« Hassid et pécheurs - le sefer ha-tokhahot d'Ezra Habavli »), Hakibbutz Hameuchad, Collection Helal Ben Haïm, 2005.

\section{ANNEXES}

שיר משל עזרא הבבלי

\begin{tabular}{|c|c|}
\hline 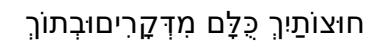 & 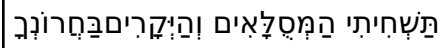 \\
\hline 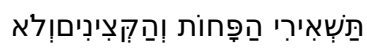 & 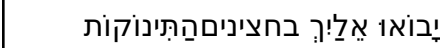 \\
\hline 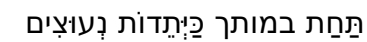 & רָּל חַכְמֵי הַלֵב וְהַיוֹעְעִצִים \\
\hline
\end{tabular}




\begin{tabular}{|c|c|}
\hline 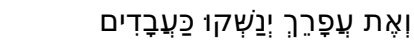 & 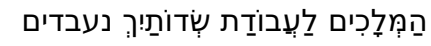 \\
\hline 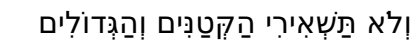 & 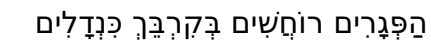 \\
\hline 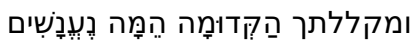 & 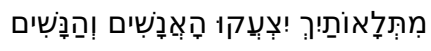 \\
\hline 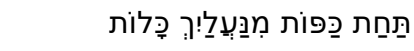 & 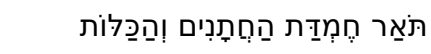 \\
\hline עָפָר יָשְׁימו בְּרֹאשָׁם & בִּמִקוֹם מבחור בְּרוֹשָׁם \\
\hline 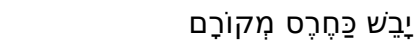 & בִּמְקוֹם הַדְרַת יְְקרָם \\
\hline 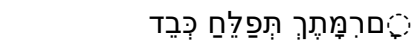 & בִִּמְקוֹם \\
\hline חְמָתָּך שוֹתָה רוּחָם & בְִּמְקוֹם מִרְקַחת רֵיחָם \\
\hline 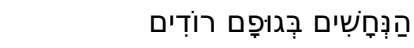 & 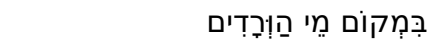 \\
\hline 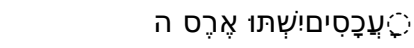 & 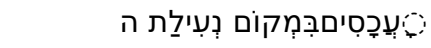 \\
\hline 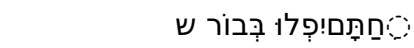 & 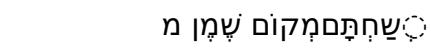 \\
\hline 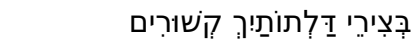 & 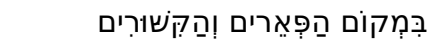 \\
\hline וּשְּׁתוּ כּוֹס הַתַּרְעַלוֹת & 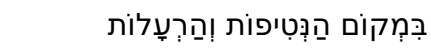 \\
\hline 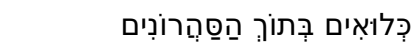 & 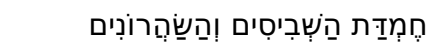 \\
\hline 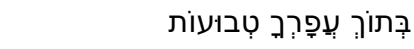 & נִזמֵמי הָאַף וְהַטַבְּעוֹת \\
\hline 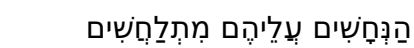 & 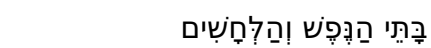 \\
\hline 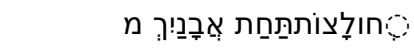 & 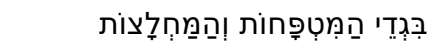 \\
\hline 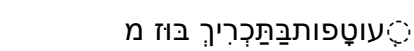 & 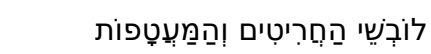 \\
\hline תכלים בְַּּּגוֹן וַאַנָחָה & 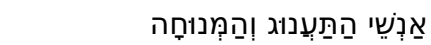 \\
\hline
\end{tabular}

\section{NOTES}

1. C'est ainsi que l'on nomme les émeutes antijuives qui ont eu lieu à Bagdad les $1^{\mathrm{er}}$ et 2 juin 1941. 


\section{RÉSUMÉS}

Histoire de la littérature hébraïque moderne chez les Juifs irakiens

Cet article traite plus de deux cent cinquante ans de littérature hébraïque moderne irakienne, poésie, prose, journaux, ainsi que la diffusion de la langue en Irak via les classes du heder et du Talmud-Torah. La première des trois parties de l'article couvre la littérature hébraïque et ses influences en Irak de 1735 à 1950. La deuxième décrit le déplacement en Israël et les difficultés rencontrées par les auteurs émigrants. La dernière section traite de la littérature hébraïque, écrite en Israël par des auteurs d'origine irakienne. Hakak conclut avec l'espoir que les lecteurs narquois et les critiques littéraires sauront finalement accorder à cette littérature la reconnaissance qu'elle mérite.

This article discusses over two hundred fifty years of modern Iraqi Hebrew literature, poetry, prose, and newspapers, as well as the dissemination of the language in Iraq via heder and Talmud-Torah classes. The first of the article's three sections covers Hebrew literature and its influences in Iraq from 1735 to 1950. The second describes the relocation to Israel and the difficulties encountered by emigrant authors. The final section discusses the Hebrew literature, including salient themes, written in Israel by authors of Iraqi descent. Hakak concludes with the wry hope that readers and literary critics will finally grant this literature the recognition it deserves.

המאמר עוסק במאתים שנות יצירה של ספרות עברית מודרנית בעיראק, בשירה, בפרוזה ובעיתונות,

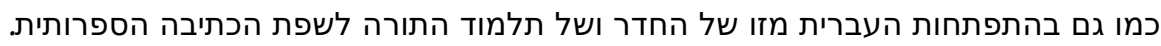

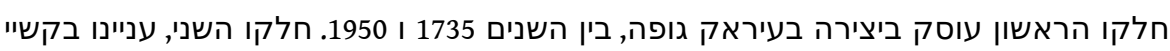

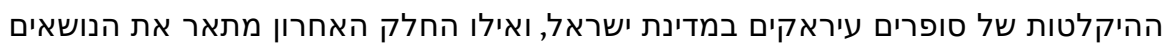

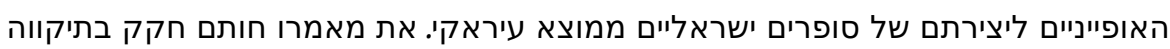

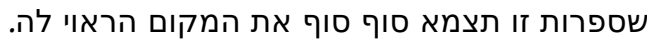

\section{INDEX}

Keywords : Hebrew literature, Iraqi Jews, piyyutim, Halakha, Hebrew poetry from Iraq, Hebrew poetry of Spain, rabbinical schools, Luzzatto Moshe Hayyim (1707-1747), eighteenth century, nineteenth century, twentieth century, Iraq, literature

Mots-clés : juifs irakiens, littérature hébraïque, piyyutim, Halakha, poésie hébraïque d'Irak, poésie hébraïque d'Espagne, écoles rabbiniques, Luzzatto Moshe Hayyim (1707-1747)

Thèmes : littérature

Index géographique : Irak

\section{מילות מפתח}

ספרות עברית, העיראקים היהודים, פיוטים, ההלכה, שירה עברית מעיראק, שירה: מילו עברית של ספרד, הרבניים בתי ספר, לוצאטו משה חיים, המאה העשרים, ספרות, עיראק Index chronologique : dix-huitième siècle, dix-neuvième siècle, vingtième siècle 